\title{
Influencia de dos aditivos en la respuesta productiva y sanitaria de cerdos en crecimiento-ceba
}

\section{Influence of two additives on the productive and sanitary response of growth-fattening pigs}

Fecha de recepción: 13 de marzo de 2016

Fecha de aprobación: 20 de octubre de 2016
Luis Gerardo Flores-Mancheno ${ }^{1}$

Yaneisy García-Hernández ${ }^{2}$

William Orlando Caicedo-Quinche ${ }^{3}$

Julio Enrique Usca-Méndez ${ }^{4}$

\section{Resumen}

El objetivo del trabajo fue estudiar la respuesta productiva y sanitaria de cerdos en crecimiento-ceba al suministro de los dos aditivos en sus dietas (preparado microbiano y antibiótico promotor del crecimiento). Se utilizaron 120 cerdos castrados de 71 días y 28,62 $\pm 2,66 \mathrm{~kg}$, distribuidos en tres grupos, según diseño completamente aleatorizado; a cada grupo se le aplicó un tratamiento, con cuatro repeticiones cada uno. Los tratamientos fueron: concentrado sin aditivo (T1); concentrado más 1,0 y 0,5 kg de virginiamicina al $2 \%$ por tonelada de alimento para cerdos en crecimiento y ceba, respectivamente (T2), y concentrado más $15 \mathrm{~mL}$ del preparado microbiano por kg de peso vivo (PV) de los animales (T3). El preparado contenía $4 \times 10^{6} \mathrm{UFC} / \mathrm{mL}$ de bacterias lácticas y $1,5 \times 10^{5} \mathrm{UFC} / \mathrm{mL}$ de levaduras. Los animales tratados con el T3 tuvieron el mayor peso final $(98,41 \mathrm{~kg}, \mathrm{P}<0,0001)$ y la mejor ganancia de peso total $(69,84 \mathrm{~kg} ; \mathrm{P}<0,0001)$ y diaria $(712,50 \mathrm{~g} / \mathrm{d}, \mathrm{P}<0,0001)$. En el mismo grupo se encontró la conversión más eficiente de materia seca $(3,04 \mathrm{~kg} / \mathrm{kg}$ PV), proteína bruta $(551,63 \mathrm{~g} / \mathrm{kg}$ PV) y energía metabolizable $(41,80 \mathrm{MJ} / \mathrm{kg}$ PV). La incidencia de diarreas fue menor en los grupos tratados con los aditivos, sin diferencia entre estos. Se concluye que los aditivos mejoran el comportamiento productivo y sanitario de cerdos en crecimiento-ceba, y que este efecto fue mayor con el preparado microbiano.

Palabras clave: antibiótico promotor de crecimiento; bacterias lácticas; crecimiento-ceba; productividad porcina; sanidad porcina.

1 Ph. D. Escuela Superior Politécnica de Chimborazo (Riobamba, Ecuador). Iflores@espoch.edu.ec.

2 Ph. D. Instituto de Ciencia Animal (Mayabeque, Cuba). yaneisyg@ica.co.cu.

3 Ph. D. Universidad Estatal Amazónica (Pastaza, Ecuador). cienciasdelatierra@uea.edu.ec.

4 M. Sc. Escuela Superior Politécnica de Chimborazo (Riobamba, Ecuador). 


\begin{abstract}
This work aimed to study the influence of two additives (microbial preparation and antibiotic growth promoter) on the productive and sanitary response of pigs in growth-feed. 120 castrated pigs of 71 days and $28.62 \mathrm{~kg}$ were used; they were distributed in three groups according to a completely randomized design with four replications each. The treatments $(\mathrm{T})$ were: $\mathrm{T} 1$, without additive; $\mathrm{T} 2$, concentrated plus 1.0 and $0.5 \mathrm{~kg}$ of $2 \%$ virginiamycin per ton of feed for growing and fattening pigs, respectively; T3, concentrated plus $15 \mathrm{~mL}$ of the microbial preparation per kg of body weight (BW) of the animal.

The preparation contained $4 \times 10^{6} \mathrm{CFU} / \mathrm{ml}$ of lactic acid bacteria and $1.5 \times 10^{5} \mathrm{CFU} / \mathrm{mL}$ of yeast. The animals treated with T3 had the highest final weight $(98.41 \mathrm{~kg}, \mathrm{P}<0.0001)$ and the best gain of total weight $(69.84 \mathrm{~kg} ; \mathrm{P}<0.0001)$ and daily gain $(712.50 \mathrm{~g} / \mathrm{d}, \mathrm{P}<0.0001)$. The most efficient conversion of dry matter (3.04 kg/kg BW), crude protein (551.63 g/ $/ \mathrm{kg} \mathrm{BW)} \mathrm{and} \mathrm{metabolizable} \mathrm{energy}(41.80 \mathrm{MJ} / \mathrm{kg} \mathrm{BW})$ was found in the same group. The incidence of diarrhea was lower in the groups treated with the additives, with no difference between them. It is concluded that the additives improve the productive and sanitary performance of pigs in growth-feed stage, and this effect was greater with the microbial preparation.
\end{abstract}

Keywords: antibiotic growth promoter; growth-feed; lactic acid bacteria; porcine productivity.

\title{
Cómo citar este artículo:
}

Flores-Mancheno LG, García-Hernández Y, Caicedo-Quinche WO, Usca-Méndez JE. Influencia de dos aditivos en la respuesta productiva y sanitaria de cerdos en crecimiento-ceba. Rev. Cien. Agri. 2017; 14(1): 65-73. 


\section{Introducción}

Los probióticos son aditivos alimentarios formados por microorganismos vivos que cuando se emplean en cantidades adecuadas afectan beneficiosamente la salud del hospedero (1). La aplicación de probióticos en la alimentación de cerdos puede contribuir a establecer el equilibrio microbiano de su tracto gastrointestinal, mejorar los procesos digestivos y absortivos, modular la respuesta inmune $y$, consecuentemente, mejorar indicadores productivos y sanitarios (2); además, estos aditivos pueden ser una alternativa a los antibióticos promotores del crecimiento (3), cuyo uso se encuentra en la actualidad restringido en varios países $(4,5)$.

Los probióticos no se emplean ampliamente en los sistemas productivos ecuatorianos, y apenas en los últimos cinco años comienzan a encontrarse trabajos investigativos donde se evidencian las ventajas de su uso $(6,7)$. En esta línea, Díaz et al. (8) informaron de la obtención de un preparado microbiano nativo a partir de suero fresco de leche, con alta concentración de bacterias lácticas y levaduras, además de ácidos orgánicos y enzimas. Este preparado fue evaluado posteriormente, a razón de $15 \mathrm{~mL} / \mathrm{kg}$ de peso vivo, en la dieta de cerdos en el período de posdestete, con efecto benéfico en su respuesta productiva y sanitaria (9); efecto que fue superior al de un probiótico con Saccharomyces cerevisiae, Bacillus subtilis y enzimas digestivas (Prince Agri Products, Inc., EUA) y al de la virginiamicina (STAFAC ${ }^{\circledR}$, Phibro, EUA), como antibiótico promotor del crecimiento animal. Sin embargo, la actividad probiótica también puede variar según la categoría animal que se emplee (10); autores como Close (11) plantearon que los resultados con probióticos parecen ser más consistentes y positivos en cerditos que en animales en la etapa de crecimiento-ceba.

Por las razones anteriores, el objetivo de la presente investigación fue estudiar la influencia de un preparado microbiano y de un antibiótico promotor del crecimiento (APC) en la respuesta productiva y sanitaria de cerdos en crecimiento-ceba.

\section{Materiales y métodos}

Localización del experimento. El experimento se desarrolló en la Unidad Académica Porcina de la Facultad de Ciencias Pecuarias (FCP) de la Escuela Superior Politécnica de Chimborazo (ESPOCH), Ecuador.

Procedimiento experimental. Se utilizaron 120 cerdos castrados, cruce Landrace-Large White $\mathrm{x}$ Belga-Pietrain, de $71 \mathrm{~d}$ de edad y con $28,62 \mathrm{~kg}$ de peso vivo (PV). Cada unidad experimental se compuso de 10 cerdos alojados en corrales colectivos de $3 \times 3,33 \mathrm{~m}$ y piso de cemento, con densidad de un cerdo por $\mathrm{m}^{2}$. Los animales se distribuyeron en tres tratamientos, con cuatro repeticiones, bajo un diseño completamente aleatorizado. El alimento balanceado se ofreció dos veces por día, a las 8:00 a.m. y 4:00 p.m. El consumo de alimento se detalla en la Tabla I. El agua se suministró ad libitum en bebederos tipo tetina.

Tabla I. Consumo de alimento promedio en la etapa de crecimiento-ceba.

\begin{tabular}{|c|c|c|}
\hline $\begin{array}{c}\text { Edad de los } \\
\text { animales (días) }\end{array}$ & $\begin{array}{c}\text { Consumo } \\
\text { promedio (kg/ } \\
\text { día) }\end{array}$ & $\begin{array}{c}\text { Consumo } \\
\text { semanal (kg) }\end{array}$ \\
\hline $71-77$ & 1,35 & 9,45 \\
\hline $78-84$ & 1,53 & 10,71 \\
\hline $85-91$ & 1,75 & 12,25 \\
\hline $92-98$ & 1,95 & 13,65 \\
\hline $99-105$ & 2,13 & 14,91 \\
\hline $106-112$ & 2,30 & 16,10 \\
\hline $113-119$ & 2,45 & 17,15 \\
\hline $120-126$ & 2,59 & 18,13 \\
\hline $127-133$ & 2,72 & 19,04 \\
\hline $134-140$ & 2,82 & 19,74 \\
\hline $141-147$ & 2,91 & 20,37 \\
\hline $148-154$ & 2,99 & 20,93 \\
\hline $155-161$ & 3,05 & 21,35 \\
\hline $162-168$ & 3,09 & 21,63 \\
\hline $\begin{array}{c}\text { Consumo total } \\
\text { de la etapa }\end{array}$ & & $\mathbf{2 3 5 , 4 1}$ \\
\hline
\end{tabular}


Los tratamientos $(\mathrm{T})$ evaluados fueron:

T1: concentrado sin aditivo

T2: concentrado + antibiótico promotor del crecimiento (virginiamicina al $2 \% ; 1,0$ y $0,5 \mathrm{~kg} / \mathrm{t}$ de alimento para cerdos en crecimiento y ceba, respectivamente)

T3: concentrado $+15 \mathrm{~mL}$ del preparado microbiano con bacterias lácticas, levaduras y enzimas (proteasas y amilasas) por kg de peso vivo.

Los alimentos balanceados se formularon según los requerimientos nutricionales de la NRC (12) para cerdos en etapa de crecimiento-ceba. Su composición se muestra en la Tabla II.

Tabla II. Composición de las dietas base utilizadas en cerdos en crecimiento-ceba.

\begin{tabular}{|l|c|c|}
\hline Ingrediente & Crecimiento & Ceba \\
\hline Maíz (\%) & 43,56 & 57,05 \\
\hline Polvillo de arroz (\%) & 7,10 & 4,30 \\
\hline Afrecho de trigo (\%) & 13,80 & 9,50 \\
\hline Harina de soya (\%) & 26,54 & 20,65 \\
\hline Harina de pescado (\%) & 0,00 & 0,20 \\
\hline DL-Metionina (\%) & 0,30 & 0,30 \\
\hline L-Lisina (\%) & 0,40 & 0,20 \\
\hline Palmiste (\%) & 0,90 & 0,50 \\
\hline Melaza (\%) & 1,80 & 2,90 \\
\hline Aceite de palma (\%) & 3,00 & 2,20 \\
\hline Carbonato de calcio (\%) & 1,60 & 1,40 \\
\hline Fosfato dicálcico (\%) & 0,20 & 0,00 \\
\hline Cloruro de sodio (\%) & 0,50 & 0,50 \\
\hline $\begin{array}{l}\text { Premezcla mineral y } \\
\left.\text { vitamínica }{ }^{1} \%\right)\end{array}$ & 0,30 & 0,30 \\
\hline Aportes calculados (90 \% de materia seca) \\
\hline Proteína bruta (\%) & 18,20 & 15,68 \\
\hline Lisina (\%) & 0,95 & 0,75 \\
\hline Metionina + Cistina (\%) & 0,54 & 0,46 \\
\hline Triptófano (\%) & 0,17 & 0,14 \\
\hline Calcio (\%) & 0,60 & 0,50 \\
\hline Fósforo (\%) & 0,60 & 0,50 \\
\hline \multicolumn{2}{|l|}{} \\
\hline
\end{tabular}

\begin{tabular}{|l|c|c|}
\hline Ingrediente & Crecimiento & Ceba \\
\hline Fósforo disponible (\%) & 0,24 & 0,21 \\
\hline Extracto etéreo (\%) & 5,80 & 5,17 \\
\hline Fibra bruta (\%) & 5,46 & 5,68 \\
\hline $\begin{array}{l}\text { Energía metabolizable } \\
\text { (MJ/kg) }\end{array}$ & 13,70 & 13,86 \\
\hline
\end{tabular}

${ }^{1}$ Pecutrín ${ }^{\circledR}(13)$

El antibiótico promotor de crecimiento (Stafac ${ }^{\circledR}$ ) se aplicó según las instrucciones del fabricante (Phibro, EUA). En el caso del preparado microbiano ecuatoriano, a base de suero de leche, se siguió para su elaboración la metodología descrita por Díaz et al. (8), y se caracterizó así: $4 \times 10^{6}$ unidades formadoras de colonia (UFC) de bacterias lácticas y $1,5 \times 10^{5}$ UFC de levaduras por cada mililitro de producto; 9,62 $\mathrm{mg} / \mathrm{L}$ de enzimas proteasas; 7,38 $\mathrm{mg} / \mathrm{L}$ de enzimas proteasas; $120 \mathrm{mg} / \mathrm{L}$ de ácido láctico, y pH de 3,9. Este preparado se aplicó en el concentrado según T3, y su cantidad se ajustó semanalmente según el peso vivo de los animales en el corral. La mezcla de ambos se realizó en recipientes de plástico para garantizar la homogeneización.

La respuesta de los cerdos al suministro de los dos aditivos en las dietas se determinó a través de los siguientes indicadores: peso vivo a los 168 días de edad; ganancia de peso de la etapa (peso final-peso inicial); ganancia de peso diaria (ganancia de peso de la etapa/días de experimentación); conversión (consumo/ganancia de peso) de materia seca (MS), proteína bruta (PB) y energía metabolizable (EM); porcentajes de animales con diarreas y porcentaje de mortalidad. También se clasificaron las diarreas producidas por Salmonella, Escherichia coli y trastornos digestivos. Para la detección de Salmonella se sembraron diariamente muestras de heces de los animales con diarreas en agar Salmonella-Shigella (14). Las placas se incubaron 18-24 $\mathrm{h}$ a $35 \pm 2^{\circ} \mathrm{C}$ y se contaron las colonias incoloras (lactosa negativa), las colonias de color rosa-rojo (lactosa-positiva) y las colonias de centro negro productoras de $\mathrm{H}_{2} \mathrm{~S}$. Para la detección de E. coli se realizaron siembras en placas Petrifilm (15). Estas se incubaron durante $24-48 \mathrm{~h}$ a $42{ }^{\circ} \mathrm{C}$ y se contaron las colonias de color azul o rojo-azul (po- 
sitivas). Las diarreas por trastornos digestivos se determinaron por diferencia entre el número total de muestras analizadas menos las que dieron positivos para Salmonella y E. coli.

Análisis estadístico. Los datos experimentales se procesaron con el paquete estadístico InfoStat (16). Se realizó análisis de varianza según diseño completamente aleatorizado y, en los casos necesarios, se aplicó la dócima de comparación de Duncan (17) para discriminar diferencias entre medias a $P<0,05$. Se aplicó un análisis de covariable para el peso vivo inicial con el objetivo de conocer si este influía en el comportamiento productivo de los animales. A las variables de conteo y a las expresadas en porcentaje (diarreas y número de muertes) se les realizó análisis de comparación de proporciones (chi cuadrado), con el paquete estadístico ComparPro (18).

\section{Resultados y discusión}

En la Tabla III se presentan los resultados del comportamiento productivo de los cerdos al aplicar los dos aditivos durante el período experimental. El peso vivo final, la ganancia de peso total, la ganancia de peso diaria y la conversión de la materia seca, proteína bruta y energía metabolizable difirieron significativamente entre tratamientos $(\mathrm{P}<0,0001)$. Solo el peso inicial, que fue una variable concomitante, no difirió. Las tres primeras variables se incrementaron con la inclusión de los aditivos en la dieta. Comportamiento que también se reflejó en la mejora de las conversiones de materia seca, proteína bruta y energía para estos tratamientos.

Tabla III. Comportamiento productivo de cerdos al incluir un antibiótico promotor del crecimiento y un preparado microbiano en la etapa de crecimiento-ceba.

\begin{tabular}{|c|c|c|c|c|}
\hline \multirow{2}{*}{ Indicador } & \multicolumn{3}{|c|}{ Tratamientos } & \multirow{2}{*}{$\begin{array}{l}\mathrm{EE}( \pm) \\
\text { signif. }\end{array}$} \\
\hline & Control & APC & $\begin{array}{c}\text { Preparado } \\
\text { microbiano }\end{array}$ & \\
\hline Peso inicial (kg) & $28,62 \pm 0,28$ & $28,71 \pm 0,35$ & $28,58 \pm 0,40$ & $\begin{array}{c}0,17 \\
P=0,8715 \\
\end{array}$ \\
\hline Peso final (kg) & $84,98^{\mathrm{a}} \pm 1,40$ & $90,28^{b} \pm 1,11$ & $98,41^{\mathrm{c}} \pm 0,57$ & $\begin{array}{c}0,54 \\
P<0,0001\end{array}$ \\
\hline Ganancia de peso total (kg) & $56,36^{\mathrm{a}} \pm 1,79$ & $61,57^{b} \pm 1,13$ & $69,84^{\mathrm{c}} \pm 0,71$ & $\begin{array}{c}0,51 \\
P<0,0001\end{array}$ \\
\hline Ganancia de peso diaria $(\mathrm{g} / \mathrm{d})$ & $575,25^{a} \pm 12,03$ & $628,50^{b} \pm 17,48$ & $712,50^{c} \pm 7,31$ & $\begin{array}{c}5,27 \\
P<0,0001 \\
\end{array}$ \\
\hline $\begin{array}{l}\text { Conversión de MS } \\
(\mathrm{kg} / \mathrm{kg} \mathrm{PV})\end{array}$ & $3,76^{c} \pm 0,07$ & $3,44^{b} \pm 0,06$ & $3,04^{a} \pm 0,03$ & $\begin{array}{c}0,03 \\
P<0,0001\end{array}$ \\
\hline $\begin{array}{l}\text { Conversión de PB } \\
\text { (g/kg PV) }\end{array}$ & $683,41^{\mathrm{c}} \pm 13,13$ & $625,46^{b} \pm 5,73$ & $551,63^{\mathrm{a}} \pm 5,84$ & $\begin{array}{c}5,54 \\
P<0,0001 \\
\end{array}$ \\
\hline Conversión de EM (MJ/kg PV) & $51,75^{c} \pm 1,04$ & $46,88^{b} \pm 0,99$ & $41,80^{a} \pm 0,44$ & $\begin{array}{c}0,40 \\
P<0,0001\end{array}$ \\
\hline
\end{tabular}

a, b, c valores con letras no comunes en la misma fila difieren a $\mathrm{P}<0,05$ (17)

Los animales que consumieron el preparado microbiano en la dieta tuvieron el mejor comportamiento productivo; con este tratamiento se obtuvo un incremento del peso final, de la ganancia de peso total y de la ganancia de peso diaria superior al control y al APC. Por consiguiente, este grupo de animales tuvo las mejores conversiones en uti- 
lización de la materia seca, la proteína bruta y la energía metabolizable.

Los incrementos anteriores y la disminución de las conversiones pudieran estar asociados, fundamentalmente, a la mejora que pueden ejercer los aditivos en los procesos de digestión y absorción de los nutrientes de la dieta, que a su vez pueden incidir en el comportamiento productivo $(19,20)$. Los animales en la etapa de crecimiento-ceba ya tienen establecido su ecosistema gastrointestinal y un sistema enzimático desarrollado, sin embargo, los antibióticos promotores del crecimiento, así como los aditivos microbianos pueden modificar la composición de este ecosistema y alterar el metabolismo microbiano y del hospedero. Por ello, quizás la obtención de una mejor respuesta productiva de los animales tratados con el preparado microbiano se relacione con la presencia de las multiespecies benéficas identificadas por Díaz et al. (8), así como con la concentración que tiene de las enzimas amilasas $(7,38 \mathrm{mg} / \mathrm{L})$ y proteasas $(9,62$ $\mathrm{mg} / \mathrm{L}$ ). Estas enzimas pudieron incidir en los procesos de digestión y, posteriormente, en una mayor absorción de nutrientes, lo que repercute en los valores de los indicadores productivos determinados.

Los resultados del presente trabajo concuerdan con los informados por otros autores, como Chen et al. (21), al emplear una mezcla de Saccharomyces cerevisiae, Lactobacillus acidophilus y BaciIlus subtilis en la dieta de cerdos en ceba; también, Choi et al. (22) y Giang et al. (23), quienes al aplicar probióticos con multicepas-multiespecies obtuvieron efectos positivos en el incremento de peso vivo, conversión alimenticia y digestibilidad de la materia seca de cerdos en ceba.

En la Figura 1 se muestra el porcentaje de cerdos en crecimiento-ceba con diarreas al suministrar el preparado microbiano y la virginiamicina como antibiótico promotor del crecimiento; en este caso se detectaron diferencias $(\mathrm{P}<0,0001)$ entre el control y los aditivos evaluados, sin diferencia entre estos últimos. Las causas de las diarreas de los cerdos también experimentaron reducción (Tabla IV); la mayor reducción (64 unidades porcentuales) se obtuvo en las diarreas causadas por Salmonella spp. para el grupo con el aditivo microbiano.

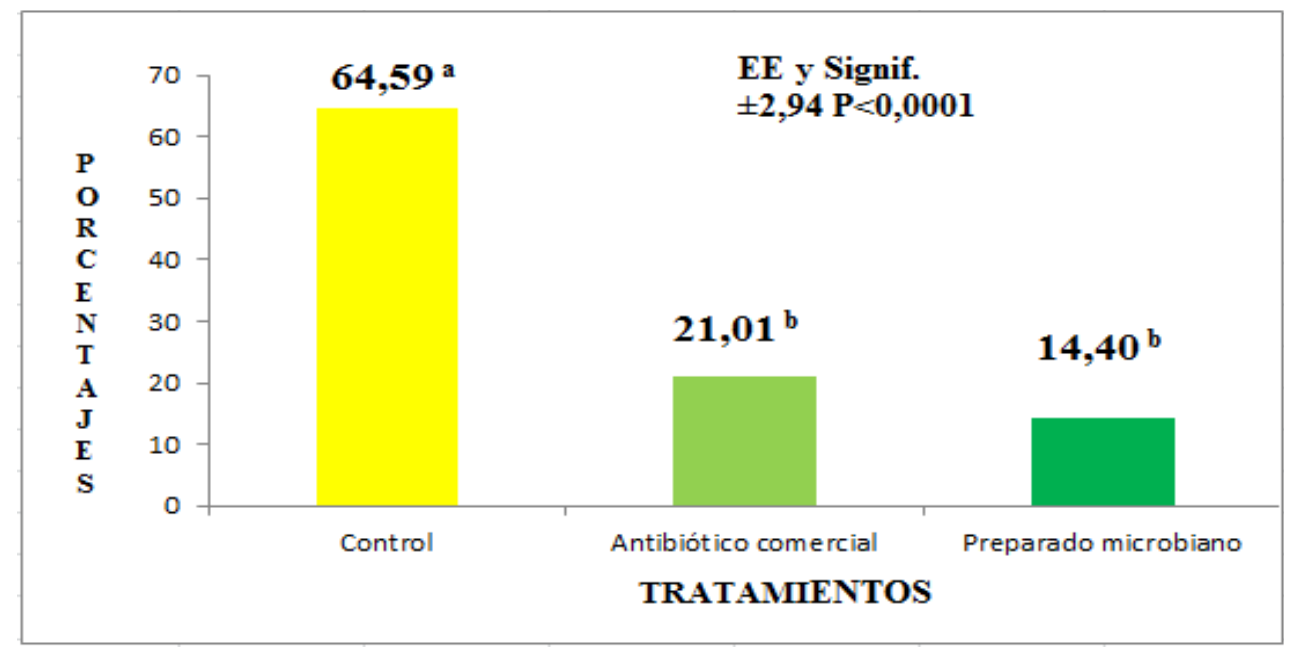

$\mathrm{a}, \mathrm{b}$ valores con letras no comunes difieren a $\mathrm{P}<0,05$ (17)

Fig. 1. Influencia del preparado microbiano y el antibiótico promotor del crecimiento en el porcentaje de cerdos en crecimiento-ceba con diarreas. 
Tabla IV. Efecto del preparado microbiano y el antibiótico promotor del crecimiento en las causas de diarreas de cerdos en crecimiento-ceba.

\begin{tabular}{|c|c|c|c|c|c|}
\hline $\begin{array}{l}\text { Causas de dia- } \\
\text { rreas }\end{array}$ & Tratamientos & $\begin{array}{l}\text { Número de anima- } \\
\text { les con diarrea }\end{array}$ & $\begin{array}{c}\% \text { de animales } \\
\text { con diarrea }\end{array}$ & $\begin{array}{l}\text { Número total } \\
\text { de animales con } \\
\text { diarrea }\end{array}$ & $\begin{array}{l}\text { EE }( \pm) \\
\text { signif. }\end{array}$ \\
\hline \multirow{3}{*}{ E. coli } & Control & 18 & $62,07^{\mathrm{a}}$ & \multirow{3}{*}{29} & \multirow{3}{*}{$\begin{array}{c}8,75 \\
P<0,0001\end{array}$} \\
\hline & APC & 7 & $24,14^{\mathrm{b}}$ & & \\
\hline & $\begin{array}{c}\text { Preparado micro- } \\
\text { biano }\end{array}$ & 4 & $13,79^{b}$ & & \\
\hline \multirow{3}{*}{$\begin{array}{l}\text { Salmonella } \\
\text { spp. }\end{array}$} & Control & 18 & $72,00^{\mathrm{a}}$ & \multirow{3}{*}{25} & \multirow{3}{*}{$\begin{array}{c}9,43 \\
P<0,0001\end{array}$} \\
\hline & APC & 5 & $20,00^{b}$ & & \\
\hline & $\begin{array}{l}\text { Preparado micro- } \\
\text { biano }\end{array}$ & 2 & $8,00^{b}$ & & \\
\hline \multirow{3}{*}{$\begin{array}{l}\text { Problemas di- } \\
\text { gestivos }\end{array}$} & Control & 130 & $64,04^{a}$ & \multirow{3}{*}{203} & \multirow{3}{*}{$\begin{array}{c}3,31 \pm \\
P<0,0001\end{array}$} \\
\hline & APC & 42 & $20,69^{b}$ & & \\
\hline & $\begin{array}{l}\text { Preparado micro- } \\
\text { biano }\end{array}$ & 31 & $15,27^{b}$ & & \\
\hline
\end{tabular}

En el actual ensayo con cerdos en crecimiento-ceba se encontró que las diarreas por problemas digestivos fueron la causa de mayor incidencia en el experimento, seguido por los problemas infecciosos ocasionados por E. coli y Salmonella spp., que se presentaron casi en igual número (Tabla IV). Independientemente de estas causas, todos los porcentajes difirieron entre el grupo control y los que incluyeron los aditivos, con los menores valores para estos últimos y sin diferencias entre ellos.

La menor incidencia de diarreas al incluir los aditivos en la dieta de los animales se puede asociar con la mejora de la salud intestinal de los animales, producida a través de diferentes mecanismos para modular la composición de la comunidad microbiana y mejorar la estructura del tracto gastrointestinal (24). Se conoce que los antibióticos promotores del crecimiento reducen las infecciones entéricas y la concentración de patógenos capaces de adherirse a la mucosa intestinal $(25,26)$, lo cual incide en la disminución de metabolitos o sustancias tóxicas que modifican la morfología de las vellosidades y el peso de los órganos digestivos, lo que de forma general afecta la salud intestinal de los animales. Por su parte, los probióticos también pueden ejercer los efectos anteriores (27), variar la composición de las poblaciones del tracto gastrointestinal sin afectar su equilibrio microbiano (28), disminuir el tiempo de duración de las diarreas en cerdos y aumentar la eficiencia de los procesos digestivos y absortivos $(26,29)$. Por ejemplo, según Wang et al. (30), los lactobacilos y sus metabolitos estimulan el peristaltismo gastrointestinal y promueven la digestibilidad aparente de los nutrientes, lo que mejora el apetito, la salud de los 
cerdos y mantiene el equilibrio microbiano de su tracto gastrointestinal.

De forma general, la respuesta productiva y sanitaria de los cerdos en crecimiento-ceba indica que el preparado microbiano adicionado en la dieta produce actividad probiótica, efecto similar al encontrado cuando se aplicó este preparado en la dieta de cerditos destetados (9).

\section{Conclusión}

Los dos aditivos evaluados (preparado microbiano y virginiamicina como antibiótico promotor del crecimiento animal) mejoran el comportamiento productivo y sanitario de cerdos en crecimiento-ceba, pero el preparado microbiano tiene un efecto mayor.

\section{Referencias}

(1) FAO/WHO (Food and Agriculture Organization of the United Nations/World Health Organization). Guidelines for the evaluation of probiotics in food. Report of a joint $\mathrm{FAO} / \mathrm{WHO}$ working group on drafting guidelines for the evaluation of probiotics in food 2002; April 30 and May 1. London Ontario, Canadá. Disponible en: <http://www.who.

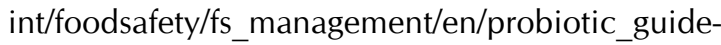
lines.pdf $>$. Consultado, enero 2017.

(2) FAO/WHO (Food and Agriculture Organization of the United Nations/World Health Organization). Health and nutritional properties of probiotics in food including powder milk with live lactic acid bacteria. Report of a joint FAO/WHO expert consultation on evaluation of health and nutritional properties of probiotics in food including powder milk with live lactic acid bacteria in food. October 1-4 2001. Córdoba, Argentina. Consultado: Abril/2015. Disponible en: < http://www.who.int/ foodsafety/ publications/fs_management/en/probiotics.pdf $>$

(3) Gutiérrez L., Montoya O., Vélez J. Probióticos: Una alternativa de producción limpia y de reemplazo a los antibióticos promotores de crecimiento en la alimentación animal. Producción + limpia. 2013; 8: 135.

(4) Maron D., Smith T., Nachman K. Restrictions on antimicrobial use in food animal production: an international regulatory and economic survey. Globalization and Health 2013; 9: 1-11. DOI: http:// doi.org/10.1186/1744-8603-9-48.

(5) Regulation EC No 1831/2003 of the European Parliament and Council of 22 September 2003 on additives for use in animal nutrition. Official J. Eur. Commun. 2003; L268: 29-43.

(6) Jurado H., Pazmiño S., Benavidez V. Evaluación del efecto probiótico de Lactobacillus plantarum en la alimentación de lechones en fase de pre ceba como una alternativa del uso de antibióticos. Rev. Investigación Pecuaria. 2013; 2: 55.

(7) Quemac M. Evaluación de tres dosis de probiótico (Rhodopseudomonas spp, Lactobacillus spp., Saccharomyces spp.) en la alimentación para el engorde de cerdos. Tesis de Ingeniería, Facultad de Veterinaria, Universidad Estatal Politécnica del Carchi, Tulcán, Ecuador. 2014.

(8) Díaz B., Elías A., Valiño E. Consorcios microbianos con actividad acido-láctica promisoria aislados desde inoculantes bacterianos nativos para ensilajes. Rev. Cien Agri. 2014; 11: 17-25. DOI: http://doi. org/10.19053/01228420.3484.

(9) Flores-Mancheno LG, García-Hernández Y., Usca-Méndez J., Caicedo-Quinche WO. Estudio comparativo de tres aditivos zootécnicos en el comportamiento productivo y sanitario de cerdos en el período post-destete. Rev. Cien. Agri. 2016; 13(2):95-105.

(10) Canibe N. Alimentación de lechones. Sistema de alimentación y aditivos en piensos de iniciación. En: XXIII Curso de especialización FEDNA. 2007; pp. 179-212.

(11) Close W. Chapter 6. Producing Pigs without Antibiotic Growth Promoters. En: Advances in Pork Production. 2000; 11: 47-56.

(12) Nutrient Requirement of Domestic Animals. Nutrient Requirements of Swine. National Academic Press.Washington, District of Columbia. 2012.

(13) Ecuaquímica. Pecutrin ${ }^{\circledR}$ saborizado. Minerales + Vitaminas A, D3, E. Suplemento mineral más vitaminas ADE. Registro: 1AB-630-AGROCALIDAD 2000; Disponible en: <http://www.ecuaquimica. com/pdf ganaderia/Pecutrin.pdf $>$ Consultado febrero 2015.

(14) Neogen. Salmonella Shigella Agar (7152) 2011; Acumedia. Disponible en: <http:// www.neogen. 
com/Acumedia/pdf/Prodlnfo/7152 Pl.pdf $>$. Consulado Enero 2017.

(15) Petrifilm 3M. Placas para recuento de E. coli/ Coliform 2010; Disponible en <http:// multimedia.3m.com/mws/media/701951O/ product-instructions-3m-petrifilm-e-coli-co liform-count-plate. pdf $>$ Consultado junio 2015.

(16) InfoStat. Di Rienzo J., Casanoves F., Balzarini M., González L., Tablada M., Robledo C. Grupo InfoStat, FCA, Universidad Nacional de Córdoba, Argentina. Versión 1.0 para Windows 2012.

(17) Duncan D. Multiple range and multiple F tests. Biometrics. 1955; 11: 1. DOI: http://doi. org/10.2307/3001478.

(18) ComparPro versión 1. Font H., Noda A., Torres V., Herrera M., Lizazo D., Sarduy L., Rodríguez L. Instituto de Ciencia Animal, Departamento de Biomatemática. 2007.

(19) Gaines AM, Allee GL, Ratliff BW, Srichana P., Nimmo RD, Gramm BR. Determination of energy value of $\operatorname{Stafac}^{\circledR}$ (virginiamycin) in finishing pigs. In: Proc. Allen D. Leman Swine Conf., St. Paul, MN. 2005; p. 35.

(20) Stewart L., Kim B., Gramm, B., Nimmo R., Stein, $H$. Effects of virginiamycim on the apparent ileal digestibility of amino acids by growing pigs. Journal of Animal Science. 2010; 88: 1718-1724. DOI: http://doi.org/10.2527/jas.2009-2063.

(21) Chen Y., Min B., Cho H., Kwon O., Son K., Kim H., Kim I. Effects of dietary Bacillus-based probiotic on growth performance, nutrient digestibility, blood characteristics and fecal noxious gas content in finishing pigs. Asian-australas. Journal of Animal Science. 2006; 19: 587-592. DOI: http://doi. org/10.5713/ajas.2006.587.

(22) Choi J., Kim J., Ingale L., Kim K., Shinde L., Kwon K., Chae B. Effect of potential multimicrobe probiotic product processed by high drying temperature and antibiotic on performance of weanling pigs. Journal of Animal Science. 2011; 89: 1795-1904. DOI: http://doi.org/10.2527/jas.2009-2794.

(23) Giang H., Viet T., Ogle B., Lindberg J. Effects of Supplementation of Probiotics on the Performance, Nutrient Digestibility and Fecal Microflora in Growing-finishing Pigs. Asian-Aust. Journal of Animal Science. 2011; 24: 655-661.

(24) Harto L., Sijtsma, R. Estrategias nutricionales para reducir la contaminación ambiental en la produc- ción de cerdos. En: XXIII Curso de especialización FEDNA. 2007.

(25) Budino F., Thomaz M., Kronka N., Nakaghi L., Tucci F., Fraga A., Scandolera A. Huaynate R. Effect of probiotic and prebiotic inclusion in weaned piglet diets on structure and ultra-structure of small intestine. Brazilian Archives of Biology and Technology. 2005; 6: 921-929. DOI: http://doi.org/10.1590/ S1516-89132005000800008.

(26) Ihara Y., Hyodo H., Sukegawa S., Murakami H., Morimatsu F. Isolation, characterization, and effect of administration in vivo, a novel probiotic strain from pig feces. Journal of Animal Science. 2013; 84: 434-441. DOI: http://doi.org/10.1111/ asj. 12020.

(27) Awad W., Ghareeb K., Abdel-Raheem S., Böhm, J. Effects of dietary inclusion of probiotic and symbiotic on growth performance, organ weights, and intestinal histomorphology of broiler chickens. Poultry Science. 2009; 88:49-56. DOI: http://doi. org/10.3382/ps.2008-00244.

(28) Recio I., López-Fandiño R. Ingredientes y productos lácteos funcionales: bases científicas de sus efectos en la salud. En: Alimentos Funcionales. Edición de la Fundación Española para la Ciencia y la Tecnología (FECYT). Madrid, España, 2005.

(29) Casey P., Garduner G., Casey G., Bradshaw B., Lawrol P., Lynch, P., Leonard F., Stanton C., Ross R., Fitzgerald GHC. 5-strain probiotic combination reduces pathogen shedding and alleviates disease signs in pigs challenged with Salmonella enterica Serovar Typhimurium. Applied and Environmental Microbiology. 2007; 73: 1858-1863. DOI: http:// doi.org/10.1128/AEM.01840-06.

(30) Wang D., Piao X., Zeng Z., Lu T., Zhang Q., Li P., Xue L., Kim W. Effects of Keratinase on Performance, Nutrient Utilization, Intestinal Morphology, Intestinal Ecology and Inflammatory Response of Weaned Piglets Fed Diets with Different Levels of Crude Protein. Asian-Aust Journal of Animal Science. 2011; 24: 1718-1728. DOI: http://doi. org/10.5713/ajas.2011.11132. 